\title{
An example of chaotic dynamics in 3D systems via stretching along paths
}

\author{
Alfonso Ruiz-Herrera • Fabio Zanolin
}

Received: 21 September 2011 / Accepted: 21 March 2012 / Published online: 11 April 2012

(C) Fondazione Annali di Matematica Pura ed Applicata and Springer-Verlag 2012

\begin{abstract}
We extend the concept of linked twist maps to a 3D setting and develop a global geometrical method to detect the presence of complex dynamics. Our approach, which is based on a recent variant of the theory of topological horseshoes, provides an analytical proof of "chaos" which does not involve small/large parameter techniques and is robust with respect to small perturbations. An application is given to a predator-prey system in $\mathbb{R}^{3}$ with a Beddington-DeAngelis functional response.
\end{abstract}

Keywords Three-dimensional predator-prey equations · Seasonal succession · Poincaré map · Complex dynamics · Topological horseshoes

Mathematics Subject Classification 92D25 · 54H20

\section{Introduction}

The celebrated theory of Smale's horseshoe [37,38] provides a very important and useful geometrical tool to detect chaotic dynamics for some classes of homeomorphisms. The horseshoe map is an elegant and simple example of a diffeomorphism acting on a square and possessing an invariant set where the given map is conjugated to a Bernoulli shift. The appearance of

Dedicated to Professor Kenneth Palmer on his 65th birthday.

The results of this paper have been presented at the conference "Non Autonomous Differential Equations, honoring Ken Palmer on the occasion of his 65th birthday" held in Ancona on June 2011.

\footnotetext{
A. Ruiz-Herrera

Department of Applied Mathematics, Facultad de Ciencias, University of Granada, 18071 Granada, Spain e-mail: alfonsoruiz@ugr.es

F. Zanolin $(\varangle)$

Dipartimento di Matematica e Informatica, Università di Udine, Via delle Scienze 206, 33100 Udine, Italy

e-mail: fabio.zanolin@uniud.it
} 
Smale's horseshoe structures in several different situations, like the restricted three body problem or the homoclinic tangles [28], has stimulated many researchers toward the investigation of situations where horseshoe-type dynamics take place (possibly in a generalized sense). In this setting, an interesting construction arises in the study of the so-called linked twist maps (indicated also as LTMs, for brevity). A linked twist map is the composition of two twist maps which act onto two overlapping annuli $\mathscr{A}_{1}$ and $\mathscr{A}_{2}$. On each annulus $\mathscr{A}_{i}$ we have a map $\phi_{i}$ which leaves invariant the boundaries and rotates points about its center by an angle that increases with the radial coordinate. The presence of chaotic dynamics occurs for $\phi_{2}^{j} \circ \phi_{1}^{k}$, with suitable choices of $j$ and $k$. LTMs were investigated by many authors, starting with Devaney [12]. Preliminary studies involving such a kind of geometry can be traced back to the works of Bowen, Braun, Easton, Thurston (see the Introduction in [12]). As pointed out by the referee, LTMs are special dynamical systems since they are one of the very few explicit examples in which complex dynamics occur on sets of positive measure. This fact has motivated the research on these maps also in connection to ergodic theory $[7,34,49]$. From the point of view of the applications, geometrical configurations associated to LTMs appear in very different contexts, like celestial mechanics [2, p. 235], [28, p. 90], motions of particles in a magnetic field, the study of diffeomorphisms of surfaces or, more recently, in the analysis of fluid mixing $[43,47]$. With this respect, the past three decades have witnessed a growing interest in this area of research. We refer to $[25,40]$ for recent contributions as well as a list of pertinent references.

The classical notion of LTMs is naturally associated to mappings acting on an invariant 2D surface. In particular, the cases of the plane, the torus, and the two-sphere have been studied in [39]. However, only few extensions of the linked twist map construction have been obtained in three or higher dimensions. See [42] for a recent contribution and also [46] for a discussion of some related geometries. In this paper, we propose an extension of this theory to dynamical systems in $\mathbb{R}^{3}$. The geometrical situation we want to discuss is that of a mapping which can be represented as the composition of two homeomorphisms $\Phi^{1}$ and $\Phi^{2}$ having the following behavior.

For $\Phi^{1}$, we assume there exists a topological cylinder $\mathscr{C}_{1}$ such that

- $\quad \mathscr{C}_{1}$ as well as its upper and lower faces $\mathscr{C}_{1}^{U}, \mathscr{C}_{1}^{L}$ are positively invariant for $\Phi^{1}$;

- $\Phi^{1}$ satisfies a twist condition with respect to $\mathscr{C}_{1}^{U}$ and $\mathscr{C}_{1}^{L}$, in the sense that, for a suitably chosen system of polar coordinates, there is a gap between the rotation numbers associated to $\left.\Phi^{1}\right|_{\mathscr{C}_{1}^{U}}$ and $\left.\Phi^{1}\right|_{\mathscr{C}_{1}^{L}}$.

For $\Phi^{2}$, we assume there exists a topological cylinder $\mathscr{C}_{2}$ such that

- $\mathscr{C}_{2}$ as well as its inner and outer boundary surfaces $\mathscr{C}_{2}^{I}, \mathscr{C}_{2}^{O}$ are positively invariant for $\Phi^{2}$

- $\Phi^{2}$ satisfies a twist condition with respect to $\mathscr{C}_{2}^{I}$ and $\mathscr{C}_{2}^{O}$, in the sense that, relatively to the axis of the cylinder, there is a gap between the rotation numbers associated to $\left.\Phi^{2}\right|_{\mathscr{C}_{2}}$ and $\left.\Phi^{2}\right|_{\mathscr{C}_{2}^{O}}$.

Under these conditions, together with additional assumptions on the intersection of $\mathscr{C}_{1}$ and $\mathscr{C}_{2}$ (see Fig. 1) as well as on the compression of $\Phi^{2}$ along some natural directions, we can prove the presence of complex dynamics for the map $\Phi^{2} \circ \Phi^{1}$ on a suitable subset of $\mathscr{C}_{1} \cap \mathscr{C}_{2}$.

The abstract topological theorems guaranteeing the presence of such chaotic-like dynamics are given in Sect. 2. A concrete example of a 3D dynamical system with this geometry is given in Sect. 3. More precisely, we consider a predator-prey model in a periodically varying environment described by an equation of the form 


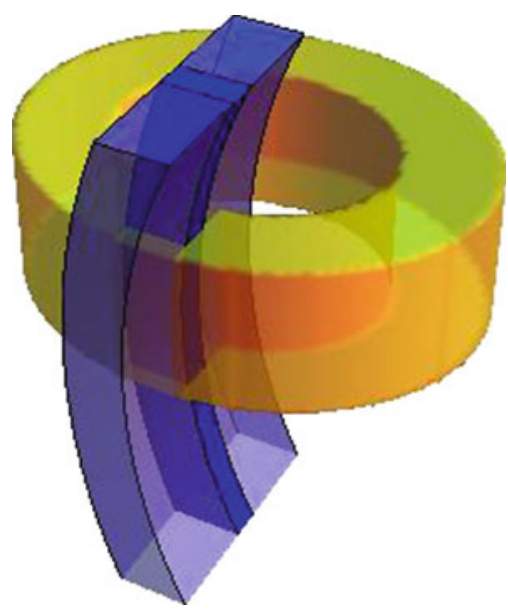

Fig. 1 The linked configuration studied in this paper. Consider two cylinders with axes of symmetry parallel to the coordinate axes $x$ and $z$, respectively, and with an intersection like in the figure

$$
\left\{\begin{array}{l}
x_{1}^{\prime}=x_{1}\left(\alpha(t)-a(t) x_{1}\right)+f_{12}\left(t, x_{1}, x_{2}, x_{3}\right) x_{2}+f_{13}\left(t, x_{1}, x_{2}, x_{3}\right) x_{3} \\
x_{2}^{\prime}=x_{2}\left(\beta(t)-b(t) x_{2}\right)-f_{21}\left(t, x_{1}, x_{2}, x_{3}\right) x_{1}+f_{23}\left(t, x_{1}, x_{2}, x_{3}\right) x_{3} \\
x_{3}^{\prime}=x_{3}\left(\gamma(t)-c(t) x_{3}\right)-f_{31}\left(t, x_{1}, x_{2}, x_{3}\right) x_{1}-f_{32}\left(t, x_{1}, x_{2}, x_{3}\right) x_{2}
\end{array}\right.
$$

where all the functions are $T$-periodic in the time variable and $a(t), b(t), c(t)$ are nonnegative. Our system is motivated by the classical results of Beddington and DeAngelis on $2 \mathrm{D}$ predator-prey system $[5,11]$ as well as to the developments of such a model in the periodic case [10]. Accordingly, the functions $f_{i j}$ are defined as

$$
f_{i j}\left(t, x_{1}, x_{2}, x_{3}\right)=\frac{a_{i j}(t) x_{i}}{1+A_{i j}(t) x_{1}+B_{i j}(t) x_{2}+C_{i j}(t) x_{3}}
$$

with $a_{i j}(t), A_{i j}(t), B_{i j}(t), C_{i j}(t) \geq 0$.

There is a broad literature dealing with chaotic dynamics for differential systems using different tools such as estimates on Lyapunov exponents, the Mel'nikov or the Sil'nikov methods, properties of ergodicity or mixing associated to some invariant measures, the Conley-Ważewki theory or some fixed point indices, just to mention a few different approaches (see, for instance $[4,14,30,35,41]$ for some significant examples and applications). Implementations of these methods to concrete ODE systems often require, from the theoretical point of view, some asymptotic estimates involving the consideration of small/large parameters or, in the applied side, to rely on computer-assisted proofs. Our approach is topological in nature and involves the study of some geometric properties of the Poincaré map associated to Eq. (1.1). The advantage of this method is that we do not require any hyperbolicity condition and we are able to impose precise lower bounds on the coefficients which are easily computable in terms of the equations. In the past 15 years, a great deal of research work has been devoted to study possible extensions of the classical Smale's horseshoe theory to a topological setting, that is keeping some important aspects of the horseshoe geometry for a given map, without using conditions on the derivatives [6,50]. Such developments of the theory of topological horseshoes have been successfully applied in the planar context or for some higher dimensional Hamiltonians (see $[1,8,32,48]$ and the references therein). Apart from the classical results of Smale and Alekseev, up to now, fewer applications in higher dimensions, especially in the non-Hamiltonian setting, like in case of (1.1), are available. 
Another meaningful property in our results is the robustness with respect to small perturbations. From the point of view of the applications, this feature is quite relevant since in the process of modeling in biology, small errors must be always considered.

\section{Topological tools}

In this section, we describe the topological tools and the notion of chaos which are used in the paper. We present our results for a discrete dynamical system determined by a homeomorphism $\Phi$ defined on a compact set $X \subset \mathbb{R}^{N}$. In our applications, $\Phi$ will be the Poincaré map associated to a first-order differential system in $\mathbb{R}^{3}$ and $X$ will be homeomorphic to a solid cylinder.

A development of the topological setting for the Smale horseshoe was originally given by Moser in his book and is based on the joint seminars of Conley and Moser at New York university in 1969-1970 [28, p. 62]. Generalizations of Moser's results to higher dimensions were widely explored in the book of Wiggins [46] as well. Our approach is closely related to the work of Kennedy and Yorke [20] with a special emphasis to the detection of periodic points, an aspect of the theory which does not fit in the general setting of metric spaces considered in [20].

We say that $\Phi$ is chaotic if there exists a compact set $\Lambda \subset X$ which is invariant for $\Phi$ (that is $\Phi(\Lambda)=\Lambda$ ) and such that $\left.\Phi\right|_{\Lambda}$ is semiconjugate to the Bernoulli shift $\sigma$ on two symbols. More precisely, there exists $g$ a continuous and surjective map so that the diagram

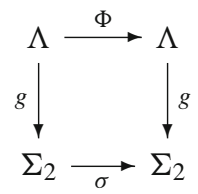

commutes. Moreover, we impose two additional conditions:

(i) If $\left(s_{i}\right)_{i \in \mathbb{Z}}$ is a $k$-periodic sequence (that is $s_{i+k}=s_{i}$ for some $k \geq 1$ ), there exists a $k$-periodic sequence $\left(w_{i}\right)_{i \in \mathbb{Z}} \in \Lambda^{\mathbb{Z}}$, satisfying $w_{i+1}=\Phi\left(w_{i}\right)$ and such that $g\left(w_{0}\right)=$ $\left(s_{i}\right)_{i \in \mathbb{Z}}$.

(ii) $\Lambda=\operatorname{cl}(\operatorname{Per} \Phi)$,

where $(\operatorname{Per} \Phi)$ is the set of periodic points of $\Phi$ in $\Lambda$.

We recall that $\Sigma_{2}=\{0,1\}^{\mathbb{Z}}$ is the set of the two-sided sequences of two symbols, with the distance

$$
d\left(\xi^{\prime}, \xi^{\prime \prime}\right):=\sum_{i=-\infty}^{+\infty} \frac{\left|s_{i}^{\prime}-s_{i}^{\prime \prime}\right|}{2^{|i|+1}}, \quad \text { for } \xi^{\prime}=\left(s_{i}^{\prime}\right)_{i}, \xi^{\prime \prime}=\left(s_{i}^{\prime \prime}\right)_{i}
$$

and the Bernoulli shift $\sigma$ is defined by

$$
\sigma: \Sigma_{2} \rightarrow \Sigma_{2}, \quad \sigma\left(\left(s_{i}\right)_{i \in \mathbb{Z}}\right)=\left(s_{i+1}\right)_{i \in \mathbb{Z}} \cdot
$$

We notice that a map which is chaotic according to our definition is also chaotic in the sense of Block and Coppel and also in the coin tossing sense [3, Remarks 3.2], [22]. As a consequence, we have that $\Phi$ has positive topological entropy (see [45] for the pertinent definitions). Besides these properties that are typically assumed as an evidence of chaotic behavior, we stress the special role played by the periodic points of $\Phi$ in our definition. We recall that just the semiconjugation with the shift map does not guarantee, in general, the 
existence of periodic points for $\Phi$ (see [9] and [20] for specific examples). In the recent years, several investigations have been devoted to the development of topological tools for proving the presence of complex dynamics accompanied by periodic points of each order. We recall [50] and [51], for some papers where similar definitions of chaos are considered.

After having defined the notion of chaos which is involved in our paper, now we introduce the main definitions and topological tools that are necessary in our approach.

By an oriented cylinder of $\mathbb{R}^{N}$ we mean a set which is the product of a closed ball in $\mathbb{R}^{N-1}$ with a compact interval and where we have put in evidence two opposite faces. Just to start with a generic example (up to homeomorphism), let us consider a cylinder of the form

$$
\mathscr{C}=B_{R} \times[0,1],
$$

where $B_{R}$ is the closed ball of center the origin and radius $R>0$ in $\mathbb{R}^{N-1}$. The base and the top of the cylinder will be denoted by $\mathscr{C}_{0}^{-}=B_{R} \times\{0\}$ and $\mathscr{C}_{1}^{-}=B_{R} \times\{1\}$. We also call an oriented cylinder the pair

$$
\widetilde{\mathscr{C}}:=\left(\mathscr{C}, \mathscr{C}^{-}\right)
$$

with

$$
\mathscr{C}^{-}:=\mathscr{C}_{0}^{-} \cup \mathscr{C}_{1}^{-}
$$

Let $K$ be a (nonempty) compact subset of $\mathscr{C}$ and let $\Phi: \mathscr{C} \rightarrow \mathbb{R}^{N}$ be a continuous map. We say that the pair $(\Phi, K)$ has the stretching along the paths property with respect to $\mathscr{C}$ and write

$$
(\Phi, K): \tilde{\mathscr{C}} \cong \tilde{\mathscr{C}},
$$

if, for every (continuous) path $\gamma:\left[t_{0}, t_{1}\right] \rightarrow \mathscr{C}$ such that $\gamma\left(t_{0}\right) \in \mathscr{C}_{0}^{-}$and $\gamma\left(t_{1}\right) \in \mathscr{C}_{1}^{-}$(or $\gamma\left(t_{0}\right) \in \mathscr{C}_{1}^{-}$and $\left.\gamma\left(t_{1}\right) \in \mathscr{C}_{0}^{-}\right)$, there exists a subinterval $\left[s_{0}, s_{1}\right]$ of $\left[t_{0}, t_{1}\right]$ for which we have that

$\gamma(t) \in K$, for all $t \in\left[s_{0}, s_{1}\right]$

$\Phi(\gamma(t)) \in \mathscr{C}$, for all $t \in\left[s_{0}, s_{1}\right]$

$\Phi\left(\gamma\left(s_{0}\right)\right)$ and $\Phi\left(\gamma\left(s_{1}\right)\right)$ belong to different components of $\mathscr{C}^{-}$.

If $K_{0}$ and $K_{1}$ are two (nonempty) compact and disjoint subsets of $\mathscr{C}$ such that

$$
\left(\Phi, K_{i}\right): \widetilde{\mathscr{C}} \cong \tilde{\mathscr{C}} \text {, for } i=0,1 \text {, }
$$

then we also write

$$
\left(\Phi, K_{0}, K_{1}\right): \tilde{\mathscr{C}} \cong \widetilde{\mathscr{C}} .
$$

The above definitions easily extend to the case in which the stretching property is satisfied between two different (oriented) cylinders.

The next theorem relates the previous concepts with our definition of chaos. Although the proof can be deduced from some results and arguments in [33], we prefer to give a self-contained proof with all the details for the reader's convenience.

Theorem 2.1 Let $\tilde{\mathscr{C}}, K_{0}$ and $K_{1}$ be as above and let $\Phi: \mathscr{C} \rightarrow \mathbb{R}^{N}$ be a homeomorphism (onto its image). Suppose that $\left(\Phi, K_{0}, K_{1}\right): \widetilde{\mathscr{C}} \cong \widetilde{\mathscr{C}}$. Then, $\Phi$ is chaotic.

Proof Preliminarily to any further discussion, we observe that it is not restrictive to assume that

$$
\Phi\left(K_{0} \cup K_{1}\right) \subset \mathscr{C}
$$


In fact, if we replace the sets $K_{0}$ and $K_{1}$ by the new compact sets $K_{0} \cap \Phi^{-1}(\mathscr{C})$ and $K_{1} \cap$ $\Phi^{-1}(\mathscr{C})$, we can easily check that the stretching along the paths property is satisfied with respect to these new sets. Therefore, from now we assume (2.1) along the proof.

First of all, we prove the existence of a fixed point for the map $\Phi$ in $K_{0}$ as well as in $K_{1}$. We shall consider, for the moment, only the case of the set $K_{0}$ (the other case is completely similar). By the Tietze extension theorem, we define a new continuous map $\tilde{\Phi}: \mathscr{C} \rightarrow \mathscr{C}$ as a continuous extension to $\mathscr{C}$ of $\left.\Phi\right|_{K_{0}}$. Our goal is to prove that $\tilde{\Phi}$ has at least a fixed point in $K_{0}$ and so a fixed point of $\Phi$ is found.

The equation $x=\tilde{\Phi}(x)$, with $x=\left(x_{1}, \ldots, x_{N}\right)$ and $\tilde{\Phi}=\left(\tilde{\Phi}_{1}, \ldots, \tilde{\Phi}_{N}\right)$, is written as a system of the form

$$
\left\{\begin{array}{l}
y-\phi(y, w)=0 \\
w-\psi(y, w)=0
\end{array}\right.
$$

where $\phi=\left(\tilde{\Phi}_{1}, \ldots, \tilde{\Phi}_{N-1}\right), \psi=\tilde{\Phi}_{N}$ and $y=\left(x_{1}, \ldots, x_{N-1}\right), w=x_{N}$.

Let $P_{R}: \mathbb{R}^{N-1} \rightarrow B_{R}$ be the radial projection onto the ball in $\mathbb{R}^{N-1}$ and define

$$
\phi^{*}(y, w):=\phi\left(P_{R}(y), w\right), \text { for all } y \in \mathbb{R}^{N-1}, w \in[0,1] .
$$

Now we take a radius $r>R$ and treat $w$ as parameter. Observe that, for each $w \in[0,1]$, all the fixed points of $\phi^{*}(\cdot, w)$ are contained in $B_{R}$. Therefore, there are no zeros of $I_{\mathbb{R}^{N-1}}-\lambda \phi^{*}(\cdot, w)$ on $\partial B_{r}$ for every $\lambda \in[0,1]$ and by standard properties of the Brouwer degree in $\mathbb{R}^{N-1}$, we conclude that

$$
\operatorname{deg}\left(I_{\mathbb{R}^{N-1}}-\phi^{*}(\cdot, w), \operatorname{int} B_{r}, 0\right)=1, \text { for all } w \in[0,1] .
$$

The Leray-Schauder Fundamental Theorem [23] (see also [26, Theorem 2.2] for a modern statement of this theorem) ensures the existence of a continuum $S \subset \operatorname{int} B_{r} \times[0,1]$ of solution pairs $(y, w)$ of the equation $y-\phi^{*}(y, w)=0$, such that $S$ projects onto $[0,1]$ along the $w$-component. By the definition of $\phi^{*}$, we have that $S \subset \mathscr{C}$ and therefore

$$
y=\phi(y, w), \quad \text { for all }(y, w) \in S .
$$

Moreover, we know that

$$
S \cap \mathscr{C}_{0}^{-} \neq \emptyset, \quad S \cap \mathscr{C}_{1}^{-} \neq \emptyset .
$$

For any $\varepsilon \in] 0, r-R$ [ we take an open $\varepsilon$-neighborhood $\mathcal{U}(\varepsilon)$ of $S$ made by a finite union of open balls of radius $\varepsilon$ centered at some points of $S$. The set $\mathcal{U}(\varepsilon)$ is open and arcwise connected. Therefore, there exists a continuous map $\gamma_{\varepsilon}:[0,1] \rightarrow \mathcal{U}(\varepsilon)$ so that, if we denote by $\gamma_{\varepsilon}^{(1)}(t)$ the vector of the first $(N-1)$-components of $\gamma_{\varepsilon}(t)$ and by $\gamma_{\varepsilon}^{(2)}(t)$ the last component of $\gamma_{\varepsilon}(t)$, we deduce that

$$
\gamma_{\varepsilon}(0) \in \mathscr{C}_{0}^{-}, \gamma_{\varepsilon}(1) \in \mathscr{C}_{1}^{-} \text {and } \gamma_{\varepsilon}^{(2)}(t) \in[0,1] \text {, for all } t \in[0,1] .
$$

The image $\gamma_{\varepsilon}([0,1])$ is not necessarily contained in $\mathscr{C}$; however, we easily obtain such a property if we replace $\gamma_{\varepsilon}(t)$ by the path

$$
\hat{\gamma}_{\varepsilon}:[0,1] \ni t \mapsto\left(P_{R}\left(\gamma_{\varepsilon}^{(1)}(t)\right), \gamma_{\varepsilon}^{(2)}(t)\right) .
$$

We can apply now the stretching along the paths assumption on $\hat{\gamma}_{\varepsilon}$ to conclude that there exists an interval $\left[s_{0}^{\prime}, s_{0}^{\prime \prime}\right] \subset[0,1]$ such that

$\hat{\gamma}_{\varepsilon}(t) \in K_{0}$, for all $t \in\left[s_{0}^{\prime}, s_{0}^{\prime \prime}\right]$

$\Phi\left(\hat{\gamma}_{\varepsilon}(t)\right) \in \mathscr{C}$, for all $t \in\left[s_{0}^{\prime}, s_{0}^{\prime \prime}\right]$; 
$\Phi\left(\hat{\gamma}_{\varepsilon}\left(s_{0}^{\prime}\right)\right)$ and $\Phi\left(\hat{\gamma}_{\varepsilon}\left(s_{0}^{\prime \prime}\right)\right)$ belong to different components of $\mathscr{C}_{0}^{-} \cup \mathscr{C}_{1}^{-}$.

Moreover, by the first of the above properties and the definition of $\tilde{\Phi}$ as an extension of $\left.\Phi\right|_{K_{0}}$, we have that $\Phi\left(\hat{\gamma}_{\varepsilon}(t)\right)=\tilde{\Phi}\left(\hat{\gamma}_{\varepsilon}(t)\right)$, for each $t \in\left[s_{0}^{\prime}, s_{0}^{\prime \prime}\right]$.

Evaluating $w-\psi(y, w)$ along the points of $\hat{\gamma}_{\varepsilon}(t)$ for $t \in\left[s_{0}^{\prime}, s_{0}^{\prime \prime}\right]$, we are lead to consider the continuous function $\left[s_{0}^{\prime}, s_{0}^{\prime \prime}\right] \ni t \mapsto \gamma_{\varepsilon}^{(2)}(t)-\psi\left(\hat{\gamma}_{\varepsilon}(t)\right)$ which is nonnegative at one side of the interval and nonpositive on the other side. Hence there exists a point $\left(y_{\varepsilon}, w_{\varepsilon}\right)=\hat{\gamma}_{\varepsilon}\left(\bar{t}_{\varepsilon}\right)$, for some $\bar{t}_{\varepsilon} \in\left[s_{0}^{\prime}, s_{0}^{\prime \prime}\right]$ such that $w_{\varepsilon}-\psi\left(y_{\varepsilon}, w_{\varepsilon}\right)=0$ with $\left(y_{\varepsilon}, w_{\varepsilon}\right) \in K_{0}$. Letting $\varepsilon \rightarrow 0^{+}$ and using the compactness of $K_{0}$, we deduce the existence of a point $\left(y^{*}, w^{*}\right) \in K_{0} \cap S$ which is a fixed point for the map $\Phi$. As already remarked at the beginning of the proof, a similar argument allows to find a fixed point for $\Phi$ in $K_{1}$.

Having proved the existence of fixed points for $\Phi$ in $K_{0}$ and in $K_{1}$, we now focus our attention on the periodic points. Let $\mathbf{s}:=\left(s_{0}, s_{1}, \ldots, s_{m}\right)$ be a $(m+1)$-tuple with $s_{i} \in\{0,1\}$ for each $i=0, \ldots, m$ and such that $s_{0}=s_{m}$. We look for a periodic point $z$ of $\Phi$ such that, setting

$$
z_{0}=z, \quad z_{i+1}=\Phi\left(z_{i}\right), \quad \text { for all } i=0, \ldots, m-1,
$$

we have

$$
z_{m}=z_{0}
$$

with

$$
z_{i} \in K_{s_{i}}, \text { for all } i=0, \ldots, m .
$$

To this end, we define the set

$$
K(\mathbf{s}):=\left\{x \in K_{s_{0}}: \Phi^{(i)}(x) \in K_{s_{i}}, \text { for all } i=1, \ldots, m\right\} .
$$

Since the stretching along the paths property is preserved by compositions,

$$
\left(\Phi^{(m)}, K(\mathbf{s})\right): \tilde{\mathscr{C}} \cong \tilde{\mathscr{C}} .
$$

Therefore, by the first part of the proof applied to $\Phi^{(m)}$ (the $m$ th iterate of $\Phi$ ), we have that there exists a fixed point $z$ of $\Phi^{(m)}$ with $z \in K(\mathbf{s})$. By the definition of $K(\mathbf{s})$ and for $s_{0}=s_{m}$, we conclude that $z$ satisfies conditions (2.3), (2.4), and (2.5).

Until now, no assumption of homeomorphism for $\Phi$ has been used; therefore, the continuity of $\Phi$ is enough to guarantee the existence of fixed points and periodic points.

We introduce now the set

$$
\mathcal{I}:=\bigcap_{i=-\infty}^{\infty} \Phi^{i}(K), \quad \text { for } K:=K_{0} \cup K_{1} .
$$

The set $\mathcal{I} \subset \mathscr{C}$ is compact and invariant for $\Phi$. A point $x$ belongs to $\mathcal{I}$ if and only if its orbit for $\Phi$ is contained in $K$. Hence, for every $x \in \mathcal{I}$, and every $i \in \mathbb{Z}$, either $\Phi^{(i)}(x) \in K_{0}$ or $\Phi^{(i)}(x) \in K_{1}$. Accordingly, to each point $x \in \mathcal{I}$ we can associate (in a unique manner) a two-sided sequence of integers

$$
g(x)=\left(s_{i}\right)_{i \in \mathbb{Z}}
$$

with $s_{i}=0$ or $s_{i}=1$, according to the fact that $\Phi^{(i)}(x) \in K_{0}$ or $\Phi^{(i)}(x) \in K_{1}$. 
By definition, the map $g: \mathcal{I} \rightarrow \Sigma_{2}$ is well defined, uniformly continuous, and the diagram

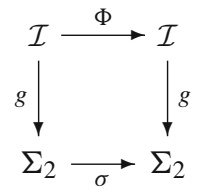

commutes. Moreover, by the previous step in the proof, for any periodic sequence $\xi=$ $\left(s_{i}\right)_{i \in \mathbb{Z}} \in \Sigma_{2}$ there exists a periodic point $z \in K_{s_{0}} \cap \mathcal{I}$ such that $g(z)=\xi$. Hence, if we denote by $\operatorname{Per}(\Phi)$ the set of periodic points for $\Phi$ in $\mathcal{I}$ and by $\operatorname{Per}(\sigma)$ the set of two-sided periodic sequences in $\{0,1\}$, we have that $g$ maps $\operatorname{Per}(\Phi)$ onto $\operatorname{Per}(\sigma)$, and, by density, $g$ is also surjective from $\mathcal{I}$ onto $\Sigma_{2}$. Finally, for

$$
\Lambda:=\operatorname{cl}(\operatorname{Per}(\Phi)),
$$

we conclude that $\Phi$ is chaotic according to our definition.

Remark 2.1 In the language of the paper of Kennedy et al. [19], the stretching along the path property is a way to express the fact that the arcs in $\mathscr{C}$ connecting $\mathscr{C}_{0}^{-}$to $\mathscr{C}_{1}^{-}$are family of expanders for $\left\{K_{0}, K_{1}\right\}$ (with respect to $\Phi$ ). Moreover, the lower and upper faces $\mathscr{C}_{0}^{-}$to $\mathscr{C}_{1}^{-}$ of our cylinder play the role of the sets $e n d_{0}$ and $e n d_{1}$ in [20] and our assumptions imply Kennedy and Yorke's horseshoe hypothesis with crossing number two. Hence, the existence of an invariant set $\mathcal{I}$ such that $\left.\Phi\right|_{\mathcal{I}}$ is semiconjugate with the shift on two symbols could be deduced from results in [19] and [20] (see also [21] for some recent extensions). On the other hand, our special configuration allows also to obtain a theorem on the existence of fixed points on $K_{0}$ and $K_{1}$ (see the first part of our proof) as well as the existence of periodic points of every order. These extra properties are, in general, not guaranteed by the above quoted papers. For a more detailed discussion of the consequences of our definition of chaos (also including sensitive dependence and transitivity), as well for a comparison with other approaches, we refer to [27, Theorem 2.2].

We remark that the assumptions in Theorem 2.1 can be suitably modified in order to produce a result which is stable with respect to small perturbations of the map $\Phi$. With this purpose, we have

Corollary 2.1 Let $\mathscr{C}, K_{0}$, and $K_{1}$ be as above and let $\Phi: \mathscr{C} \rightarrow \mathbb{R}^{N}$ be a homeomorphism (onto its image). Suppose that $\left(\Phi, K_{0}, K_{1}\right): \widetilde{\mathscr{C}} \cong \widetilde{\mathscr{C}}$, with

$$
\left.K_{0}, K_{1} \subset B_{R} \times\right] 0,1\left[\text { and } \Phi\left(K_{0}\right) \cap \mathscr{C}, \Phi\left(K_{1}\right) \cap \mathscr{C} \subset \operatorname{int} B_{R} \times[0,1] .\right.
$$

Then, there exists $\varepsilon>0$ such that every homeomorphism $\Psi: \mathscr{C} \rightarrow \Psi(\mathscr{C})$ is chaotic, provided that

$$
\|\Psi(x)-\Phi(x)\| \leq \varepsilon, \text { for all } x \in \mathscr{C} .
$$

Theorem 2.1 and Corollary 2.1 are stable by homeomorphisms deforming the domain.

\section{Chaotic dynamics in 3D predator-prey systems}

Motivated by the classical predator-prey model with Beddington-DeAngelis functional response (see $[5,10,11,29]$ ), we consider a system of the form

$$
\left\{\begin{array}{l}
x_{1}^{\prime}=x_{1}\left(\alpha(t)-a(t) x_{1}\right)+f_{12}\left(t, x_{1}, x_{2}, x_{3}\right) x_{2}+f_{13}\left(t, x_{1}, x_{2}, x_{3}\right) x_{3} \\
x_{2}^{\prime}=x_{2}\left(\beta(t)-b(t) x_{2}\right)-f_{21}\left(t, x_{1}, x_{2}, x_{3}\right) x_{1}+f_{23}\left(t, x_{1}, x_{2}, x_{3}\right) x_{3} \\
x_{3}^{\prime}=x_{3}\left(\gamma(t)-c(t) x_{3}\right)-f_{31}\left(t, x_{1}, x_{2}, x_{3}\right) x_{1}-f_{32}\left(t, x_{1}, x_{2}, x_{3}\right) x_{2}
\end{array}\right.
$$


where all the functions are $T$-periodic in the time variable, $a(t), b(t), c(t)$ are nonnegative, and the functions $f_{i j}$ are defined as

$$
f_{i j}\left(t, x_{1}, x_{2}, x_{3}\right)=\frac{a_{i j}(t) x_{i}}{1+A_{i j}(t) x_{1}+B_{i j}(t) x_{2}+C_{i j}(t) x_{3}}
$$

with $a_{i j}(t), A_{i j}(t), B_{i j}(t), C_{i j}(t) \geq 0$. From the proof, we will see that our results can be easily adapted to the cases when in system (3.1) the nonlinearities are like those considered in [36, p. 224] or [31, p. 248].

From a biological point of view, the previous system describes the evolution of three species sharing the same seasonally varying environment. The behavior of each species can be summarized in the following way:

- The species $x_{1}$ is a predator for $x_{3}$ and $x_{2}$.

- The species $x_{2}$ is a prey for $x_{1}$ and a predator for $x_{3}$.

- The species $x_{3}$ is a prey for $x_{1}$ and $x_{2}$.

In many biological situations, the seasonal dependence is expressed as a switching between two or more regimes, where each regime is described by an autonomous system. In population dynamics as well as in epidemiology, such kind of equations have been widely studied and are known as systems with seasonal succession $[13,15,16]$. For instance, these systems naturally appear when the behavior of some species alternates between different states (like migration, hibernation, or breeding periods), see [13,15-18,24].

In this framework, a particular case of (3.1) is given by the $T$-periodic differential equation defined as

$$
\begin{aligned}
& \left\{\begin{array} { l } 
{ x _ { 1 } ^ { \prime } = x _ { 1 } ( - \alpha _ { 1 } + a _ { 1 2 } \frac { x _ { 2 } } { 1 + x _ { 3 } } ) } \\
{ x _ { 2 } ^ { \prime } = x _ { 2 } ( \beta _ { 1 } - a _ { 2 1 } x _ { 1 } ) } \\
{ x _ { 3 } ^ { \prime } = 0 }
\end{array} \quad \text { for all } t \in \left[n T, n T+T_{1}[\right.\right. \\
& \left\{\begin{array} { l } 
{ x _ { 1 } ^ { \prime } = x _ { 1 } ( - \alpha _ { 2 } + a _ { 1 3 } x _ { 3 } ) } \\
{ x _ { 2 } ^ { \prime } = x _ { 2 } ( \beta _ { 2 } - x _ { 2 } ) } \\
{ x _ { 3 } ^ { \prime } = x _ { 3 } ( \gamma _ { 2 } - a _ { 3 1 } x _ { 1 } ) }
\end{array} \quad \text { for all } t \in \left[n T+T_{1},(n+1) T[,\right.\right.
\end{aligned}
$$

where all the parameters are strictly positive. For convenience, we introduce the notation $(\mathbf{S})$ to denote the above $T$-periodic system made by (3.2)-(3.3) and set

$$
T_{2}:=T-T_{1} .
$$

It is easy to check that system (S) is a particular case of (3.1). For instance, $\alpha(t)$ is the $T$-periodic function defined as $-\alpha_{1}$ if $t \in\left[0, T_{1}\right.$ [ and as $-\alpha_{2}$ if $t \in\left[T_{1}, T[\right.$.

Our goal is to illustrate how the construction presented in the Introduction appears in (S) and so in (3.1). With this respect, the following results hold.

Proposition 3.1 Fix all the parameters in (S) except $\gamma_{2}$ and assume that

$$
0<\beta_{2}<\left(1+\frac{\alpha_{2}}{2 a_{13}}\right) \frac{\alpha_{1}}{a_{12}} .
$$

Then, there exist $\gamma_{2}^{*}, T_{1}^{*}$, and $T_{2}^{*}\left(\gamma_{2}\right)$ so that if $\gamma_{2}>\gamma_{2}^{*}, T_{1}>T_{1}^{*}$, and $T_{2}>T_{2}^{*}\left(\gamma_{2}\right)$, the Poincaré map associated to $(\mathbf{S})$ is chaotic.

Remark 3.1 In the proof, we estimate $\gamma_{2}^{*}, T_{1}^{*}$, and $T_{2}^{*}\left(\gamma_{2}\right)$ depending on the coefficients of the system. 
Theorem 3.1 Fix all the parameters in (S) verifying the conditions of Proposition 3.1, i.e., assume (3.4) and $\gamma_{2}>\gamma_{2}^{*}, T_{1}>T_{1}^{*}, T_{2}>T_{2}^{*}\left(\gamma_{2}\right)$. Then, there exists $\epsilon>0$ such that if the distance in $L_{T}^{1}$ between the previous parameters in $(\mathbf{S})$ and the coefficients of (3.1) is smaller than $\epsilon$, the Poincaré map associated to (3.1) is chaotic.

Given two $T$-periodic integrable functions $f(t)$ and $g(t)$, their distance in $L_{T}^{1}$ is given by $\int_{0}^{T}|f(t)-g(t)| d t$. In our setting, the $\epsilon$-assumptions in Theorem 3.1 mean that

$$
\int_{0}^{T_{1}}\left|\alpha(t)-a_{1}\right| d t+\int_{T_{1}}^{T}\left|\alpha(t)-a_{2}\right| d t<\epsilon,
$$

and so on (for the other coefficients). Recall that the Poincaré map is defined as

$$
\begin{aligned}
& \Phi: \mathbb{R}_{+}^{3} \longrightarrow \mathbb{R}_{+}^{3} \\
& p \mapsto x(T, p),
\end{aligned}
$$

where $x(t, p)$ is the maximal solution of the system with initial condition at $p$. We have employed the notation

$$
\mathbb{R}_{+}^{3}=\left\{\left(x_{1}, x_{2}, x_{3}\right): x_{i} \geq 0, \text { for } i=1,2,3\right\} .
$$

We observe that the functions satisfying conditions of form (3.5) are not necessary "piecewise constant" and they can be taken as smooth as we like.

\section{Proofs}

\subsection{Proof of Proposition 3.1}

We split the proof of Proposition 3.1 into four steps. More precisely, we start by presenting some preliminary results on the classical Lotka-Volterra model in the planar case. Secondly, we build the 3D regions needed in our construction of linked cylinders and introduce a parallelepiped where we apply the topological results of Sect. 2. Finally, we check the validity of the stretching along the path conditions by studying the Poincaré maps associated to $(\mathbf{S})$.

Step 1: Geometric properties of the classical planar Lotka-Volterra system.

As a first step, we give two geometric lemmas concerning the trajectories of the system

$$
\left\{\begin{array}{l}
x_{1}^{\prime}=x_{1}\left(-A+B x_{2}\right) \\
x_{2}^{\prime}=x_{2}\left(C-D x_{1}\right) .
\end{array}\right.
$$

Recall that all the nontrivial orbits of this system are closed curves surrounding the equilibrium point $(C / D, A / B)$. These curves have the expression

$$
\mathcal{E}\left(x_{1}, x_{2}\right)=k>\min \mathcal{E}=\mathcal{E}(C / D, A / B),
$$

for the energy function

$$
\mathcal{E}\left(x_{1}, x_{2}\right):=D x_{1}-C \log x_{1}+B x_{2}-A \log x_{2} .
$$

In the sequel, when we write $\mathcal{E}_{\alpha}(\cdot, \cdot)$, for $\alpha$ one of the coefficients $A, B, C, D$, we mean the energy $\mathcal{E}$ defined above in which we allow $\alpha$ to vary and keep all the other three coefficients fixed. 


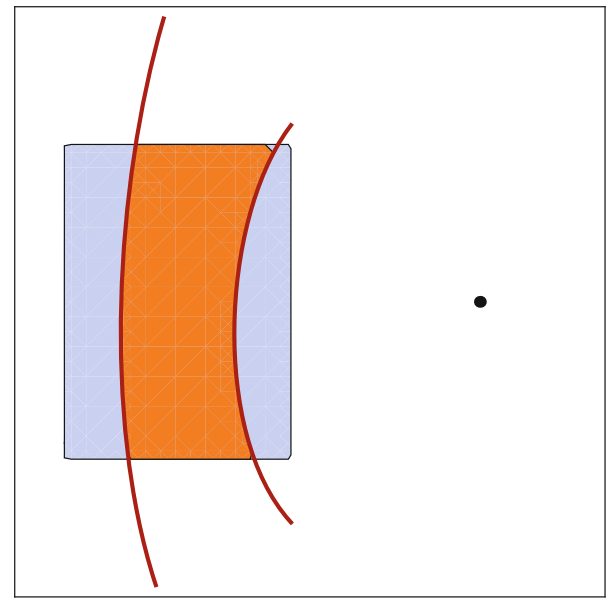

Fig. 2 An illustration of Lemma 4.1

Lemma 4.1 Consider system (4.1) with A, B, D fixed positive parameters and the rectangle

$$
S=\left[x_{0}-l_{1}, x_{0}+l_{1}\right] \times\left[\frac{A}{B}-l_{2}, \frac{A}{B}+l_{2}\right] \subset \operatorname{int} \mathbb{R}_{+}^{2}
$$

for $x_{0}, l_{1}, l_{2}$ satisfying $0<l_{1}<x_{0}$ and $0<l_{2}<\frac{A}{B}$. Then, there exists a constant $\Omega>$ $D\left(x_{0}+l_{1}\right)$ such that for every $C>\Omega$, we have that solutions $(x(t), y(t))$ of system (4.1) with initial conditions at $\left(x_{0}-\frac{l_{1}}{2}, \frac{A}{B}\right)$ and at $\left(x_{0}+\frac{l_{1}}{2}, \frac{A}{B}\right)$ leave the rectangle $S$ across the sides $\left[x_{0}-l_{1}, x_{0}+l_{1}\right] \times\left\{\frac{A}{B}-l_{2}\right\}$ and $\left[x_{0}-l_{1}, x_{0}+l_{1}\right] \times\left\{\frac{A}{B}+l_{2}\right\}$.

A possible lower bound for $\Omega$ is given by $\left(x_{0}+l_{1}\right)\left(D+\frac{4 B^{2} l_{2}^{2}}{\left(A-B l_{2}\right) l_{1}}\right)$.

Proof As long as $\left(x_{1}, x_{2}\right) \in S$, we have the following estimate

$$
\left|\frac{d x_{1}}{d x_{2}}\right|=\left|\frac{x_{1}\left(-A+B x_{2}\right)}{x_{2}\left(C-D x_{1}\right)}\right| \leq \frac{\left|x_{0}+l_{1}\right| B l_{2}}{\left|\frac{A}{B}-l_{2}\right|\left(C-D\left(x_{0}+l_{1}\right)\right)}=K .
$$

Now it is clear that if $2 \mathrm{Kl}_{2}<\frac{l_{1}}{2}$, the property of the lemma holds.

Figure 2 illustrates the previous fact.

Lemma 4.2 Consider system (4.1) with A, C, D fixed positive parameters and suppose that $B \in\left[\Gamma_{1}, \Gamma_{2}\right]$ with $0<\Gamma_{1}<\Gamma_{2}$. Given $y_{0}$, l satisfying $0<y_{0}-l<y_{0}+l$ and $\Gamma_{2}\left(y_{0}+l\right)<$ $A$, there exists $\widehat{\epsilon}>0$ so that for all $0<\epsilon \leq \widehat{\epsilon}$ we have that the solution $(x(t), y(t))$ of system (4.1) with initial condition at $\left(\frac{C}{D}, y_{0}+\frac{3 l}{4}\right)$ (resp. at $\left(\frac{C}{D}, y_{0}-\frac{3 l}{4}\right)$ ) leaves the rectangle $\left[\frac{C}{D}-\epsilon, \frac{C}{D}+\epsilon\right] \times\left[y_{0}+\frac{l}{2}, y_{0}+l\right]$ (resp. the rectangle $\left.\left[\frac{C}{D}-\epsilon, \frac{C}{D}+\epsilon\right] \times\left[y_{0}-l, y_{0}-\frac{l}{2}\right]\right)$, across the sides $\left\{\frac{C}{D}-\epsilon\right\} \times\left[y_{0}+\frac{l}{2}, y_{0}+l\right]$ and $\left\{\frac{C}{D}+\epsilon\right\} \times\left[y_{0}+\frac{l}{2}, y_{0}+l\right]$ (across the sides $\left\{\frac{C}{D}-\epsilon\right\} \times\left[y_{0}-l, y_{0}-\frac{l}{2}\right]$ and $\left.\left\{\frac{C}{D}+\epsilon\right\} \times\left[y_{0}-l, y_{0}-\frac{l}{2}\right]\right)$.

We can take $\widehat{\epsilon}=\min \left\{\sqrt{\frac{l C\left(A-\Gamma_{2}\left(y_{0}+l\right)\right)}{16 D^{2}\left(y_{0+l}\right)}}, \frac{C}{2 D}\right\}$. 
Proof The proof is totally analogous to the previous one and we omit it.

Step 2: Construction of the topological parallelepiped.

In this step, we consider system (S), namely

$$
\left\{\begin{array} { l } 
{ x _ { 1 } ^ { \prime } = x _ { 1 } ( - \alpha _ { 1 } + a _ { 1 2 } \frac { x _ { 2 } } { 1 + x _ { 3 } } ) } \\
{ x _ { 2 } ^ { \prime } = x _ { 2 } ( \beta _ { 1 } - a _ { 2 1 } x _ { 1 } ) } \\
{ x _ { 3 } ^ { \prime } = 0 }
\end{array} \quad \text { for } t \in \left[0, T_{1}[\right.\right.
$$

and

$$
\left\{\begin{array} { l } 
{ x _ { 1 } ^ { \prime } = x _ { 1 } ( - \alpha _ { 2 } + a _ { 1 3 } x _ { 3 } ) } \\
{ x _ { 2 } ^ { \prime } = x _ { 2 } ( \beta _ { 2 } - x _ { 2 } ) } \\
{ x _ { 3 } ^ { \prime } = x _ { 3 } ( \gamma _ { 2 } - a _ { 3 1 } x _ { 1 } ) }
\end{array} \quad \text { for } t \in \left[T_{1}, T[.\right.\right.
$$

The dynamics of this system can be described in the following way. We follow the trajectories of (4.2) for $t \in\left[0, T_{1}\right.$ [, those of (4.3) for $t \in\left[T_{1}, T\right.$ [, and then we repeat the same transition in a $T$-periodical manner. We are interested only in solutions starting from points in the interior of the first octant. Note that following the orbits of (4.3) along the interval $\left[T_{1}, T\right]$ is the same as following such orbits for $t \in\left[0, T_{2}\right]$. Accordingly, if we denote respectively by $\Phi^{1}$ and $\Phi^{2}$ the Poincaré maps for system (4.2) (from $t=0$ to $t=T_{1}$ ) and for system (4.3) (from $t=0$ to $t=T_{2}$ ), the Poincaré map (after time $T$ ) for the whole system is given by

$$
\Phi:=\Phi^{2} \circ \Phi^{1} \text {. }
$$

Observe that $\Phi^{1}$ and $\Phi^{2}$ (and hence also $\Phi$ ) are well defined on int $\mathbb{R}_{+}^{3}$.

Once these comments have been done, we properly start the construction of the parallelepiped. The set of the equilibrium points in int $\mathbb{R}_{+}^{3}$ for system (4.2) is given by the curve

$$
L:=\left\{\left(\frac{\beta_{1}}{a_{21}}, \frac{\alpha_{1}\left(1+x_{3}\right)}{a_{12}}, x_{3}\right): x_{3}>0\right\} .
$$

Every horizontal plane $x_{3}=$ constant $>0$ is invariant for the solutions of system (4.2), and on this plane, we have a classical Lotka-Volterra predator-prey equation with a global center at the point $\left(\frac{\beta_{1}}{a_{21}}, \frac{\alpha_{1}\left(1+x_{3}^{0}\right)}{a_{12}}\right)$. Therefore, for any point $q_{0}:=\left(x_{1}^{0}, x_{2}^{0}, x_{3}^{0}\right) \notin L$ and for any $\tau>0$, we can define a rotation number in the following way:

$$
\operatorname{rot}\left(q_{0}, \tau\right):=\frac{1}{2 \pi} \int_{0}^{\tau} \frac{\left(x_{2}\left(t ; q_{0}\right)-\frac{\alpha_{1}\left(1+x_{3}^{0}\right)}{a_{12}}\right) X_{1}(t)-\left(x_{1}\left(t ; q_{0}\right)-\frac{\beta_{1}}{a_{21}}\right) X_{2}(t)}{\left(x_{1}\left(t ; q_{0}\right)-\frac{\beta_{1}}{a_{21}}\right)^{2}+\left(x_{2}\left(t ; q_{0}\right)-\frac{\alpha_{1}\left(1+x_{3}^{0}\right)}{a_{12}}\right)^{2}} d t,
$$

where

$$
X_{1}(t):=x_{1}\left(t ; q_{0}\right)\left(-\alpha_{1}+a_{12} \frac{x_{2}\left(t ; q_{0}\right)}{1+x_{3}^{0}}\right), \quad X_{2}(t):=x_{2}\left(t ; q_{0}\right)\left(\beta_{1}-a_{21} x_{1}\left(t ; q_{0}\right)\right)
$$

and $\left(x_{1}\left(t ; q_{0}\right), x_{2}\left(t ; q_{0}\right), x_{3}^{0}\right)$ is the solution of system (4.2) departing at time $t=0$ from the initial point $q_{0}$.

The rotation number counts the number of winds around the equilibrium point in the plane $x_{3}=x_{3}^{0}$ of the solution $\left(x_{1}\left(t ; q_{0}\right), x_{2}\left(t ; q_{0}\right), x_{3}^{0}\right)$ along the time interval $[0, \tau]$ in the clockwise sense. Since the trajectories of (4.2) wind clockwise, $\operatorname{rot}\left(q_{0}, \tau\right)$ is a strictly increasing function of $\tau$ for any $q_{0} \notin L$. Moreover, if we denote by $P\left(q_{0}\right)$ the fundamental period of the periodic solution $\left(x_{1}\left(t ; q_{0}\right), x_{2}\left(t ; q_{0}\right), x_{3}^{0}\right)$ of $(4.2)$, we have that for a positive integer $m$,

$$
\operatorname{rot}\left(q_{0}, \tau\right) \lessgtr m \Longleftrightarrow \tau \lessgtr m P\left(q_{0}\right)
$$


holds. Next we study the dependence on the period of the orbits with respect to $x_{2}^{0}, x_{3}^{0}$ and so, by the previous comments, the dependence on the rotation number with respect to these variables. To this end, we use a result of Waldvogel [44] about the monotonicity of the period as a function of the energy for the Lotka-Volterra systems. In order to enter into the setting of [44], we make a change of variables and move the equilibrium point of the system

$$
\left\{\begin{array}{l}
x_{1}^{\prime}=x_{1}\left(-\alpha_{1}+a_{12} \frac{x_{2}}{1+x_{3}^{0}}\right) \\
x_{2}^{\prime}=x_{2}\left(\beta_{1}-a_{21} x_{1}\right)
\end{array}\right.
$$

to the new point $(1,1)$. More precisely, setting

$$
y_{1}:=\frac{a_{21}}{\beta_{1}} x_{1}, \quad y_{2}:=\frac{a_{12}}{\alpha_{1}\left(1+x_{3}^{0}\right)} x_{2},
$$

we transform (4.4) into the equivalent system

$$
\left\{\begin{array}{l}
y_{1}^{\prime}=-\alpha_{1} y_{1}\left(1-y_{2}\right) \\
y_{2}^{\prime}=\beta_{1} y_{2}\left(1-y_{1}\right)
\end{array}\right.
$$

The solution of (4.4) starting at the point $\left(\beta_{1} / a_{21}, x_{2}^{0}\right)$ corresponds to that of system (4.5) starting at the point $\left(1, a_{12} x_{2}^{0} / \alpha_{1}\left(1+x_{3}^{0}\right)\right)$ and, obviously, their period is the same. The energy of such a solution $\left(y_{1}(t), y_{2}(t)\right)$ of $(4.5)$ is

$$
h=h(Y):=\beta_{1}+a_{12} Y-\alpha_{1} \log \left(a_{12} Y / \alpha_{1}\right), \text { for } Y:=x_{2}^{0} /\left(1+x_{3}^{0}\right) .
$$

An easy computation shows that $h(Y)$ is strictly increasing for $Y>\frac{\alpha_{1}}{a_{12}}$ and strictly decreasing for $Y<\frac{\alpha_{1}}{a_{12}}$. At this moment, we can use [44, Theorem 2] which guarantees that the period function for system (4.5) is strictly monotone with respect to $h$ and, from this fact and using (3.4), we can conclude that for

$$
0<\tilde{l}<\left(1+\frac{\alpha_{2}}{2 a_{13}}\right) \frac{\alpha_{1}}{a_{12}}-\beta_{2}, \quad \tilde{l}<\frac{\beta_{2}}{2}, \quad \tilde{l}<\frac{\beta_{2} \alpha_{2}}{2\left(a_{13}+\alpha_{2}\right)},
$$

the rectangle

$$
S:=\left[\beta_{2}-\tilde{l}, \beta_{2}+\tilde{l}\right] \times\left[\alpha_{2} / 2 a_{13}, 3 \alpha_{2} / 2 a_{13}\right]
$$

has the following properties.

(i) $S \subset\left\{\left(x_{2}, x_{3}\right): \alpha_{1}>a_{12} \frac{x_{2}}{1+x_{3}}\right\}$,

$$
\underbrace{\min _{x_{2}^{0} \in\left[\beta_{2}-\tilde{l}, \beta_{2}+\tilde{l}\right]} P\left(\beta_{1} / a_{21}, x_{2}^{0}, 3 \alpha_{2} / 2 a_{13}\right)}_{P_{*}}>\overbrace{\max _{x_{2}^{0} \in\left[\beta_{2}-\tilde{l}, \beta_{2}+\tilde{l}\right]} P\left(\beta_{1} / a_{21}, x_{2}^{0}, \alpha_{2} / 2 a_{13}\right)}^{P^{*}} .
$$

The first property about $S$ is clear from the choice of the parameter $\tilde{l}$. By the same choice, we also deduce that $\min \left\{x_{2}^{0} /\left(1+x_{3}^{0}\right): x_{2}^{0} \in\left[\beta_{2}-\tilde{l}, \beta_{2}+\tilde{l}\right], x_{3}^{0}=\alpha_{2} / 2 a_{13}\right\}>\max \left\{x_{2}^{0} /(1+\right.$ $\left.\left.x_{3}^{0}\right): x_{2}^{0} \in\left[\beta_{2}-\tilde{l}, \beta_{2}+\tilde{l}\right], x_{3}^{0}=3 \alpha_{2} / 2 a_{13}\right\}$ and this yields $\left.i i\right)$ using Waldvogel theorem and the fact that the energy is decreasing on $Y$ for $Y<\alpha_{1} / a_{12}$. After these preliminary remarks, we can introduce the cylindrical set

$$
C y l:=\bigcup_{x_{2}^{0} \in\left[\beta_{2}-l_{1}, \beta_{2}+l_{1}\right]}\left\{\left(x_{1}, x_{2}, x_{3}\right): x_{3} \in\left[\lambda_{1}, \lambda_{2}\right], \mathcal{E}_{x_{3}}\left(x_{1}, x_{2}\right)=\mathcal{E}_{x_{3}}\left(\beta_{1} / a_{21}, x_{2}^{0}\right)\right\},
$$




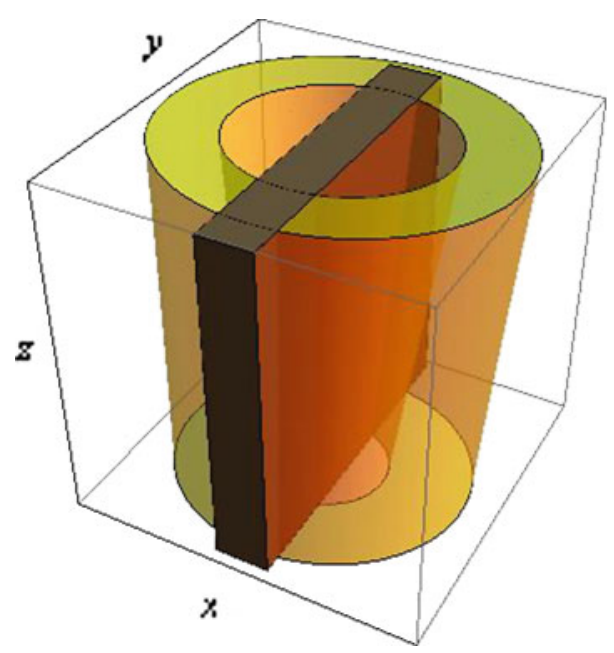

Fig. 3 The section of the cylinder $C y l$ with the resulting block $\mathscr{B}_{\hat{\varepsilon}}$

for

$$
l_{1}:=\frac{3}{4} \tilde{l}, \quad \lambda_{1}:=\frac{\alpha_{2}}{2 a_{13}}, \quad \lambda_{2}:=\frac{3 \alpha_{2}}{2 a_{13}} .
$$

The set $C y l$ lies between the horizontal planes $x_{3}=\lambda_{1}$ and $x_{3}=\lambda_{2}$. If we cut $C y l$ with a plane $x_{3}=x_{3}^{0} \in\left[\lambda_{1}, \lambda_{2}\right]$, we obtain an annular region which is filled by all the periodic orbits of system (4.4) passing through the interval of initial points $\left\{\left(\beta_{1} / a_{21}, x_{2}^{0}\right)\right.$ : for $x_{2}^{0} \in$ $\left.\left[\beta_{2}-l_{1}, \beta_{2}+l_{1}\right]\right\}$. The condition $\left.i i\right)$ says that every trajectory of system (4.2) that departs form a point of the upper face $C y l \cap\left\{x_{3}=\lambda_{2}\right\}$ of $C y l$ has a period which is strictly larger than the period of an arbitrary trajectory departing from the lower face $C y l \cap\left\{x_{3}=\lambda_{1}\right\}$. We also notice that $C y l$, as well as all its slices $C y l \cap\left\{x_{3}=\right.$ constant $\}$, is an invariant set under system (4.2).

From the cylindrical set just defined, we select now a vertical block which is the part of $C y l$ lying between two vertical planes parallel to the plane $x_{1}=\beta_{1} / a_{21}$. Namely, we define the set

$$
B_{\hat{\varepsilon}}:=\left\{\left(x_{1}, x_{2}, x_{3}\right) \in C y l: x_{1} \in\left[\left(\beta_{1} / a_{21}\right)-\hat{\varepsilon},\left(\beta_{1} / a_{21}\right)+\hat{\varepsilon}\right]\right\}
$$

where $\hat{\varepsilon}$ comes from Lemma 4.2 for the parameters $A=\alpha_{1}, B \in\left[\frac{a_{12}}{1+3 \alpha_{2} / 2 a_{13}}, \frac{a_{12}}{1+\alpha_{2} / 2 a_{13}}\right], C=\beta_{1}, D=a_{21}, y_{0}=\beta_{2}$, and $l=\widetilde{l}$ (see Fig. 3).

By construction, $B_{\hat{\varepsilon}}$ has exactly two connected components. Between these two components, we take the one which contains the rectangle

$$
\left\{\left(\beta_{1} / a_{21}, x_{2}, x_{3}\right): x_{2} \in\left[\beta_{2}-l_{1}, \beta_{2}+l_{1}\right], x_{3} \in\left[\lambda_{1}, \lambda_{2}\right]\right\}
$$

and call it $\mathscr{B}_{\hat{\varepsilon}}$. Finally, using again Lemma 4.2 we find inside the set $\mathscr{B}_{\hat{\varepsilon}}$ a parallelepiped of the form (see Fig. 4)

$$
\mathscr{R}:=\left[\left(\beta_{1} / a_{21}\right)-\hat{\varepsilon},\left(\beta_{1} / a_{21}\right)+\hat{\varepsilon}\right] \times\left[\beta_{2}-(\tilde{l} / 2), \beta_{2}+(\tilde{l} / 2)\right] \times\left[\lambda_{1}, \lambda_{2}\right] .
$$

Our goal will be to prove that the cylinder $C y l$ contains a horseshoe for $\Phi$ with suitable times $T_{1}$ and $T_{2}$ using the stretching property for the paths. In this proof, the set $\mathscr{R}$ will be 


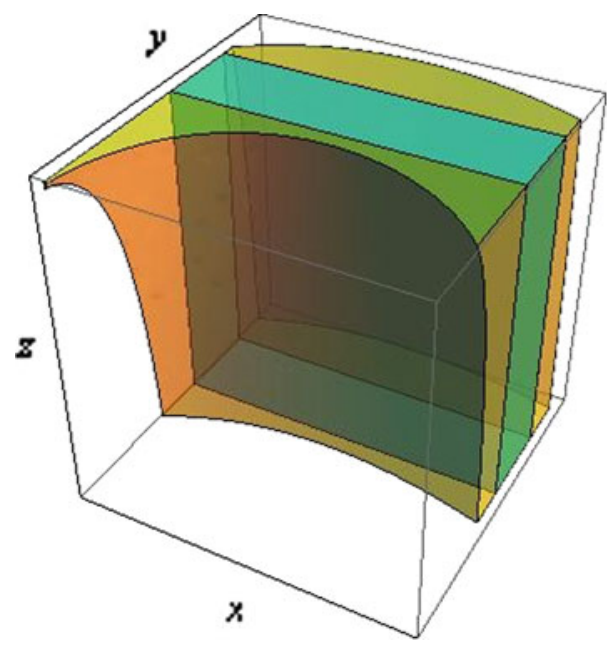

Fig. 4 The parallelepiped $\mathscr{R}$ inside the chosen component of $\mathscr{B} \hat{\varepsilon}$

involved too. Notice that all the introduced parameters in the proof can be exactly computed depending on the coefficients of the system and they do not depend on $\gamma_{2}$.

Step 3: Stretching property for system (4.2).

First of all, we give a name to the lower and upper sides of $\mathscr{B}_{\hat{\varepsilon}}$ and also select two lateral faces of such a set. Namely, we define

$$
\mathscr{B}_{0}^{-}:=\mathscr{B}_{\hat{\varepsilon}} \cap\left\{\left(x_{1}, x_{2}, x_{3}\right): x_{3}=\lambda_{1}\right\}, \quad \mathscr{B}_{1}^{-}:=\mathscr{B}_{\hat{\varepsilon}} \cap\left\{\left(x_{1}, x_{2}, x_{3}\right): x_{3}=\lambda_{2}\right\}
$$

and

$\mathscr{F}_{-}:=\mathscr{B}_{\hat{\varepsilon}} \cap\left\{\left(x_{1}, x_{2}, x_{3}\right): x_{1}=\frac{\beta_{1}}{a_{21}}-\hat{\varepsilon}\right\}, \quad \mathscr{F}_{+}:=\mathscr{B}_{\hat{\varepsilon}} \cap\left\{\left(x_{1}, x_{2}, x_{3}\right): x_{1}=\frac{\beta_{1}}{a_{21}}+\hat{\varepsilon}\right\}$.

In this step, we determine a lower bound for $T_{1}$ and prove that there exist two compact sets $H_{k_{1}}, H_{k_{2}} \subset \mathscr{B}_{\hat{\varepsilon}}$ so that

$$
\left(\Phi^{1}, H_{k_{1}}, H_{k_{2}}\right): \widetilde{\mathscr{B}_{\hat{\varepsilon}}} \cong \widetilde{\mathscr{F}}
$$

where $\widetilde{\mathscr{B}}_{\hat{\varepsilon}}=\left(\mathscr{B}_{\hat{\varepsilon}}, \mathscr{B}^{-}\right)$with $\mathscr{B}_{\hat{\varepsilon}}^{-}=\mathscr{B}_{0}^{-} \cup \mathscr{B}_{1}^{-}$and $\widetilde{\mathscr{F}}=\left(\mathscr{F}, \mathscr{F}^{-}\right)$with $\mathscr{F}=\mathscr{B}_{\hat{\varepsilon}}$, $\mathscr{F}^{-}=\mathscr{F}_{-} \cup \mathscr{F}_{+}$.

By the construction of $\mathscr{B}_{\hat{\varepsilon}}$, we know that

$$
P\left(q_{0}\right) \geq P_{*}, \text { for all } q_{0} \in \mathscr{B}_{1}^{-}, \quad P\left(q_{1}\right) \leq P^{*}, \quad \text { for all } q_{1} \in \mathscr{B}_{0}^{-} .
$$

On each plane $x_{3}=x_{3}^{0}$, with $x_{3}^{0} \in\left[\lambda_{1}, \lambda_{2}\right]$, we introduce a system of polar coordinates with center at $\left(\beta_{1} / a_{21}, \alpha_{1}\left(1+x_{3}^{0}\right) / a_{12}\right)$ and count the angles in the clockwise sense starting from the half-line $\left\{\left(\beta_{1} / a_{21}, x_{2}\right): x_{2} \leq \alpha_{1}\left(1+x_{3}^{0}\right) / a_{12}\right\}$. Each section of $\mathscr{B}_{\hat{\varepsilon}}$ with the plane $x_{3}=x_{3}^{0}$ lies in the angular region between $-\pi / 2$ and $\pi / 2$. Moreover, if we denote by $\theta(\tau, q)$ the angular coordinate at time $\tau>0$ of the solution of (4.2) departing from $q \in \mathscr{B}_{\hat{\varepsilon}}$, we deduce

$$
\theta(\tau, q)=\theta(0, q)+2 \pi \operatorname{rot}(q, \tau) \in[2 \pi \operatorname{rot}(q, \tau)-\pi / 2,2 \pi \operatorname{rot}(q, \tau)+\pi / 2] .
$$


Fix

$$
T_{1} \geq \frac{5 P_{*} P^{*}}{P_{*}-P^{*}} .
$$

Let $m_{*}=m_{*}\left(T_{1}\right)$ be the least integer such that

$$
m_{*} \geq \frac{T_{1}}{P_{*}}
$$

Then

$$
\operatorname{rot}\left(q_{0}, T_{1}\right) \leq m_{*}, \quad \text { for all } q_{0} \in \mathscr{B}_{1}^{-} .
$$

On the other hand, if we choose $m^{*}=m^{*}\left(T_{1}\right)$ the largest integer such that

$$
m^{*} \leq \frac{T_{1}}{P^{*}},
$$

then we have

$$
\operatorname{rot}\left(q_{1}, T_{1}\right) \geq m^{*}, \quad \text { for all } q_{1} \in \mathscr{B}_{0}^{-} .
$$

Moreover, from the choice of $T_{1}$ in (4.7), we know that

$$
m^{*}-m_{*} \geq 3 .
$$

As a consequence of (4.6), we also obtain

$$
\theta\left(T_{1}, q_{0}\right) \leq 2 m_{*} \pi+\pi / 2, \quad \text { for all } q_{0} \in \mathscr{B}_{1}^{-}
$$

and

$$
\theta\left(T_{1}, q_{1}\right) \geq 2 m^{*} \pi-\pi / 2, \quad \text { for all } q_{1} \in \mathscr{B}_{0}^{-} .
$$

By the above estimates, for each positive integer $k$ with

$$
k \in\left[m_{*}+1, m^{*}-1\right],
$$

the following inclusion

$$
\left[2 k \pi-\frac{\pi}{2}, 2 k \pi+\frac{\pi}{2}\right] \subset\left[\max _{q_{0} \in \mathscr{B}_{1}^{-}} \theta\left(T_{1}, q_{0}\right), \min _{q_{1} \in \mathscr{B}_{0}^{-}} \theta\left(T_{1}, q_{1}\right)\right]
$$

holds. At last, for any integer $k$ satisfying (4.8), let us define the set

$$
H_{k}:=\left\{q \in B_{\hat{\varepsilon}}: \theta\left(T_{1}, q_{0}\right) \in\left[2 k \pi-\frac{\pi}{2}, 2 k \pi+\frac{\pi}{2}\right]\right\} .
$$

Observe that, by the continuity of the angular function with respect to the initial points and the above interval inclusion, we find that the sets $H_{k}$ 's are nonempty, compact, and pairwise disjoint.

Let

$$
\gamma:[0,1] \rightarrow \mathscr{B}_{\hat{\varepsilon}}
$$

be a continuous map, with $\gamma(s)=\left(\gamma_{1}(s), \gamma_{2}(s), \gamma_{3}(s)\right)$, such that

$$
\gamma(0) \in \mathscr{B}_{0}^{-} \quad \text { and } \quad \gamma(1) \in \mathscr{B}_{1}^{-}
$$


and consider the new path

$$
[0,1] \ni s \mapsto \Phi^{1}(\gamma(s)),
$$

which represents the evolution of the points of $\gamma(s)$ after the time $T_{1}$ under the dynamical system associated to (4.2). Notice that

$$
\Phi^{1}(\gamma(s)) \in C y l, \text { for all } s \in[0,1]
$$

and, for $\Phi^{1}(\gamma(s))=\left(\Phi_{1}^{1}\left(\gamma(s), \Phi_{2}^{1}\left(\gamma(s), \Phi_{3}^{1}(\gamma(s))\right.\right.\right.$, we have $\Phi_{3}^{1}(\gamma(s))=\gamma_{3}(s)$, for all $s \in[0,1]$.

We claim that for every integer $k$ satisfying (4.8) there is an interval $\left[s_{0}, s_{1}\right]=\left[s_{0}^{k}, s_{1}^{k}\right] \subset$ $[0,1]$ such that

$$
\begin{aligned}
& \gamma(s) \in H_{k}, \quad \text { for all } s \in\left[s_{0}, s_{1}\right], \\
& \Phi^{1}(\gamma(s)) \in \mathscr{B}_{\hat{\varepsilon}}, \quad \text { for all } s \in\left[s_{0}, s_{1}\right]
\end{aligned}
$$

and

$$
\Phi^{1}\left(\gamma\left(s_{0}\right)\right) \in \mathscr{F}_{+}, \quad \Phi^{1}\left(\gamma\left(s_{1}\right)\right) \in \mathscr{F}_{-} .
$$

Indeed, recalling that $\theta\left(T_{1}, \gamma(s)\right)$ is the angular coordinate at time $T_{1}$ of the solution of (4.2) departing from $\gamma(s)$, we deduce

$\theta\left(T_{1}, \gamma(0)\right) \leq 2 m_{*} \pi+\pi / 2<2 k \pi-\pi / 2<2 k \pi+\pi / 2<2 m^{*} \pi-\pi / 2 \leq \theta\left(T_{1}, \gamma(1)\right)$.

Consequently, we can find an interval $\left[\sigma_{0}, \sigma_{1}\right] \subset[0,1]$ such that

$$
\theta\left(T_{1}, \gamma\left(\sigma_{0}\right)\right)=-\frac{\pi}{2}+2 k \pi, \quad \theta\left(T_{1}, \gamma\left(\sigma_{1}\right)\right)=\frac{\pi}{2}+2 k \pi,
$$

for the given integer $k$. Moreover, by continuity, we can also assume that

$$
-\frac{\pi}{2}+2 k \pi \leq \theta\left(T_{1}, \gamma\left(\sigma_{0}\right)\right) \leq \frac{\pi}{2}+2 k \pi, \text { for all } s \in\left[\sigma_{0}, \sigma_{1}\right] .
$$

Hence $\gamma(s) \in H_{k}$ for all $s \in\left[\sigma_{0}, \sigma_{1}\right]$. Having found $\left[\sigma_{0}, \sigma_{1}\right]$ we easily get $\left[s_{0}, s_{1}\right] \subset\left[\sigma_{0}, \sigma_{1}\right]$ as claimed, just by observing that the set is $\mathscr{B}_{\hat{\varepsilon}}$ is (on each horizontal section) angularly bounded by $[-\pi / 2, \pi / 2]$.

Step 4: Stretching property for system (4.3).

In this step, we prove that there exist $\gamma_{2}^{*}$ and $T_{2}^{*}\left(\gamma_{2}\right)$ so that for all $\gamma_{2}>\gamma_{2}^{*}$ and $T_{2}>T_{2}^{*}\left(\gamma_{2}\right)$ the following stretching property holds. Given a continuous path $\zeta:\left[s_{0}, s_{1}\right] \longrightarrow \mathscr{B}_{\hat{\varepsilon}}$ with $\zeta(0) \in \mathscr{F}_{-}$and $\zeta(1) \in \mathscr{F}_{+}$, there exists a subinterval $\left[s_{0}^{\prime \prime}, s_{1}^{\prime \prime}\right] \subset[0,1]$ so that

$$
\Phi^{2}\left(\zeta\left(\left[s_{0}^{\prime \prime}, s_{1}^{\prime \prime}\right]\right)\right) \subset \mathscr{B}_{\hat{\varepsilon}}
$$

with

$$
\begin{aligned}
& \Phi^{2}\left(\gamma\left(s_{0}^{\prime \prime}\right)\right) \in \mathscr{B}_{1}^{-} \\
& \Phi^{2}\left(\gamma\left(s_{1}^{\prime \prime}\right)\right) \in \mathscr{B}_{0}^{-} .
\end{aligned}
$$

Indeed, take a continuous path $\zeta:\left[s_{0}, s_{1}\right] \longrightarrow \mathscr{B}_{\hat{\varepsilon}}$ with $\zeta(0) \in \mathscr{F}_{-}$and $\zeta(1) \in \mathscr{F}_{+}$. First of all, we notice that, by the construction of $\mathscr{B}_{\hat{\varepsilon}}$ (which, we recall, involved the use of Lemma 4.2 with $l=\widetilde{l}$ ) there exists a constant $\kappa>0$ so that $\left|x_{2}-\beta_{2}\right| \leq \kappa$ for every point $\left(x_{1}, x_{2}, x_{3}\right) \in \mathscr{B}_{\hat{\varepsilon}}$. Thus, in particular,

$$
\left|\zeta_{2}(s)-\beta_{2}\right| \leq \kappa, \text { for all } s \in\left[s_{0}, s_{1}\right]
$$


and, from the second equation in (4.3) we easily find a precise time estimate, say $T^{*}(\tilde{l})$, such that for $T_{2} \geq T^{*}(\tilde{l})$ it holds that

$$
\left|\Phi_{2}^{2}(\zeta(s))-\beta_{2}\right| \leq \frac{\tilde{l}}{2}, \text { for all } s \in\left[s_{0}, s_{1}\right],
$$

where $\Phi^{2}=\left(\Phi_{1}^{2}, \Phi_{2}^{2}, \Phi_{3}^{2}\right)$. Having settled the behavior of $\Phi^{2}$ in its second component, we can forget for a moment the second equation in (4.3) and study the reduced subsystem

$$
\left\{\begin{array}{l}
x_{1}^{\prime}=x_{1}\left(-\alpha_{2}+a_{13} x_{3}\right) \\
x_{3}^{\prime}=x_{3}\left(\gamma_{2}-a_{31} x_{1}\right),
\end{array}\right.
$$

For this system, we apply Lemma 4.1 with the parameters:

$$
A:=\alpha_{2}, B:=a_{13}, C:=\gamma_{2}, D:=a_{31}, x_{0}=\frac{\beta_{1}}{a_{21}}, l_{1}:=\hat{\varepsilon}, l_{2}:=\frac{\alpha_{2}}{2 a_{13}}
$$

and the rectangle $S$ of Lemma 4.1 takes the form

$$
\mathcal{S}:=\left[\left(\beta_{1} / a_{21}\right)-\hat{\varepsilon},\left(\beta_{1} / a_{21}\right)+\hat{\varepsilon}\right] \times\left[\lambda_{1}, \lambda_{2}\right] .
$$

Accordingly, we can determine a constant $\Omega$ such that for

$$
\gamma_{2}>\Omega
$$

the conclusion of Lemma 4.1 holds. Recall that $\hat{\varepsilon}$ does not depend on $\gamma_{2}$. In our setting, this means that each solution of (4.9) with initial point on the segment $\left[\left(\beta_{1} / a_{21}\right)-\right.$ $\left.(\hat{\varepsilon} / 2),\left(\beta_{1} / a_{21}\right)+(\hat{\varepsilon} / 2)\right] \times\left\{\alpha_{2} / a_{13}\right\}$ leaves the rectangle $\mathcal{S}$ across the sides $\left[\left(\beta_{1} / a_{21}\right)-\right.$ $\left.\hat{\varepsilon},\left(\beta_{1} / a_{21}\right)+\hat{\varepsilon}\right] \times\left\{\lambda_{1}\right\}$ (in backward time) and $\left[\left(\beta_{1} / a_{21}\right)-\hat{\varepsilon},\left(\beta_{1} / a_{21}\right)+\hat{\varepsilon}\right] \times\left\{\lambda_{2}\right\}$ (in forward time).

By the well-known phase-portrait of the Lotka-Volterra equation, in the $x_{1} x_{3}$-plane we have a global center which is the equilibrium point $\left(\frac{\gamma_{2}}{a_{31}}, \frac{\alpha_{2}}{a_{13}}\right)$ surrounded by the energy level lines

$$
\mathcal{F}\left(x_{1}, x_{3}\right)=k>\min \mathcal{F}=\mathcal{F}\left(\gamma_{2} / a_{31}, \alpha_{2} / a_{31}\right),
$$

where

$$
\mathcal{F}\left(x_{1}, x_{3}\right):=a_{31} x_{1}-\gamma_{2} \log x_{1}+a_{13} x_{3}-\alpha_{2} \log x_{3}
$$

is the associated energy. Then, we can introduce polar coordinates and define a rotation number (as we did before in the planes $x_{3}=$ constant). More in detail, we take as a center the equilibrium point $\left(\gamma_{2} / a_{31}, \alpha_{2} / a_{13}\right)$ and we count the angles in the clockwise sense starting from the half-line $\left\{\left(x_{1}, \alpha_{2} / a_{13}\right): x_{1} \leq \gamma_{2} / a_{31}\right\}$. In particular, associate to any initial point $q:=\left(x_{1}^{0}, x_{3}^{0}\right)$, with $x_{1}^{0}<\gamma_{2} / a_{31}$ an initial angle between $-\pi / 2$ and $\pi / 2$ and denote by $\vartheta(\tau, q)$ the angle performed at the time $\tau>0$ by the solution of (4.9) departing from $q$. Note that also the rectangle $\mathcal{S}$ lies in the angular region between $-\pi / 2$ and $\pi / 2$.

We call $Q_{*}$ and $Q^{*}$ the period of the orbits of (4.9) passing through the points $\left(\left(\beta_{1} / a_{21}\right)-\right.$ $\left.(\hat{\varepsilon} / 2), \alpha_{2} / a_{13}\right)$ and $\left(\left(\beta_{1} / a_{21}\right)+(\hat{\varepsilon} / 2), \alpha_{2} / a_{13}\right)$, respectively. By the strict monotonicity of the time-map with respect to the energy levels of the orbits, we know that

$$
Q_{*}>Q^{*} .
$$

It is important to observe that for any initial point $q_{0}=\left(x_{1}^{0}, x_{2}^{0}, x_{3}^{0}\right)$, with

$$
\mathcal{F}\left(x_{1}^{0}, x_{3}^{0}\right)=k_{*}:=\mathcal{F}\left(\left(\beta_{1} / a_{21}\right)-(\hat{\varepsilon} / 2), \alpha_{2} / a_{13}\right),
$$




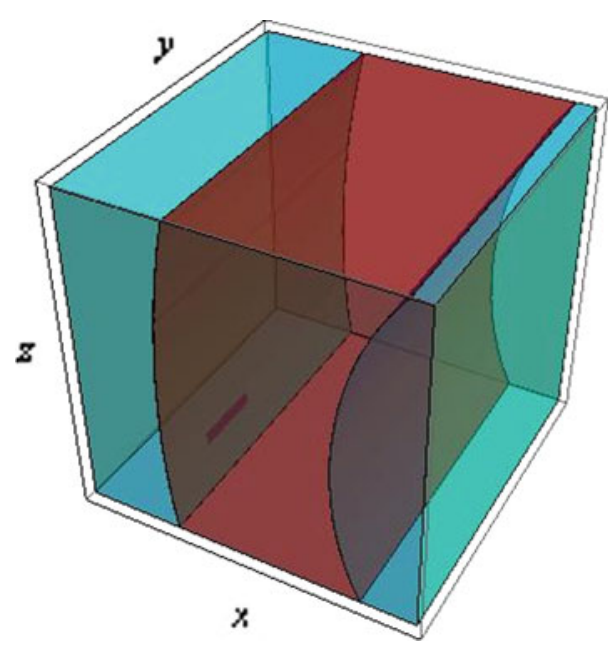

Fig. 5 The part of the region in $\mathscr{R}$ between the surfaces $\mathscr{F}=k_{*}$ and $\mathscr{F}=k^{*}$

the corresponding solution $\left(x_{1}\left(t ; q_{0}\right), x_{2}\left(t ; q_{0}\right), x_{3}\left(t ; q_{0}\right)\right)$ of $(4.3)$ lies on the invariant surface

$$
\left\{\left(x_{1}, x_{2}, x_{3}\right): \mathcal{F}\left(x_{1}, x_{3}\right)=k_{*}\right\} .
$$

The projection of such a solution on the $x_{1} x_{3}$-plane is a periodic orbit for (4.9) of period $Q_{*}$ winding around the equilibrium point $\left(a_{31} / \gamma_{2}, \alpha_{2} / a_{31}\right)$. In the meantime, the second component $x_{2}\left(t ; q_{0}\right)$ moves toward $c_{2}$ and, as we have already seen, $\left|x_{2}\left(t ; q_{0}\right)-\beta_{2}\right| \leq \tilde{l} / 2$ for $t \geq T^{*}(\tilde{l})$. A completely similar behavior occurs for the solutions of (4.3) with an initial point satisfying

$$
\mathcal{F}\left(x_{1}^{0}, x_{3}^{0}\right)=k^{*}:=\mathcal{F}\left(\left(\beta_{1} / a_{21}\right)+(\hat{\varepsilon} / 2), \alpha_{2} / a_{13}\right) .
$$

These solutions lie on the surface

$$
\left\{\left(x_{1}, x_{2}, x_{3}\right): \mathcal{F}\left(x_{1}, x_{3}\right)=k^{*}\right\}
$$

and their projection onto the $x_{1} x_{3}$-plane are periodic orbits of (4.9) with period $Q^{*}$.

Hence, by (4.10), the path $\zeta$ considered at the beginning must cross the energy lines $\mathscr{F}=k_{*}$ and $\mathscr{F}=k^{*}$, which according to Lemma 4.1 separates the left and the right sides of the rectangle $\mathcal{S}$. Figure 5 shows the domain limited by the surfaces $\mathscr{F}=k_{*}$ and $\mathscr{F}=k^{*}$ in $\mathscr{R}$.

Then, we can find a subinterval $\left[s_{0}^{\prime}, s_{1}^{\prime}\right] \subset\left[s_{0}, s_{1}\right]$ such that

$$
\mathcal{F}\left(\zeta\left(s_{0}^{\prime}\right)\right)=k^{*}, \mathcal{F}\left(\zeta\left(s_{1}^{\prime}\right)\right)=k_{*}, \quad \text { and } \mathcal{F}(\zeta(s)) \in\left[k^{*}, k_{*}\right] \text {, for all } s \in\left[s_{0}^{\prime}, s_{1}^{\prime}\right] \text {. }
$$

Fix

$$
T_{2}^{*} \geq \frac{4 Q_{*} Q^{*}}{Q_{*}-Q^{*}}
$$

We proceed now like in the previous step and define $\ell_{*}$ as the least integer larger than or equal to $T_{2}^{*} / Q_{*}$ and $\ell^{*}$ as the greater integer less than or equal to $T_{2}^{*} / Q^{*}$. By the choice of $T_{2}^{*}$ we find that $\ell^{*}-\ell_{*} \geq 2$, and therefore, for any integer $j \in\left[\ell_{*}+1, \ell^{*}-1\right]$, we have $\vartheta\left(T_{2}^{*}, \zeta\left(s_{0}^{\prime}\right)\right) \leq 2 \ell_{*} \pi+\pi / 2<2 j \pi-\pi / 2<2 j \pi+\pi / 2<2 \ell^{*} \pi-\pi / 2 \leq \vartheta\left(T_{2}^{*}, \zeta\left(s_{1}^{\prime}\right)\right)$. 
Since the set $\left\{\vartheta\left(T_{2}^{*}, \zeta(s)\right): s \in\left[s_{0}^{\prime}, s_{1}^{\prime}\right]\right\}$ covers the angular interval $[2 \pi j-\pi / 2,2 \pi j+\pi / 2]$ and also recalling that the rectangle $\mathcal{S}$ is angularly bounded in the same region, we find a subinterval $\left[s_{0}^{\prime \prime}, s_{1}^{\prime \prime}\right] \subset\left[s_{0}^{\prime}, s_{1}^{\prime}\right]$ such that

$$
\Phi_{3}^{2}\left(\zeta\left(s_{1}^{\prime \prime}\right)\right)=\lambda_{1}, \Phi_{3}^{2}\left(\zeta\left(s_{0}^{\prime \prime}\right)\right)=\lambda_{2}, \Phi_{3}^{2}(\zeta(s)) \in\left[\lambda_{1}, \lambda_{2}\right], \text { for all } s \in\left[s_{0}^{\prime \prime}, s_{1}^{\prime \prime}\right]
$$

and also

$$
\Phi_{1}^{2}(\zeta(s)) \in\left[\beta_{1} / a_{21}-\hat{\varepsilon}, \beta_{1} / a_{21}+\hat{\varepsilon}\right], \text { for all } s \in\left[s_{0}^{\prime \prime}, s_{1}^{\prime \prime}\right] .
$$

Finally, we fix

$$
T_{2} \geq \max \left\{T^{*}(\tilde{l}), T_{2}^{*}\right\}
$$

By summarizing the preceding steps, we have obtained the following: There exist two compact sets $H_{k_{1}}, H_{k_{2}} \subset \mathscr{B}_{\hat{\varepsilon}}$ so that

$$
\left(\Phi=\Phi_{2} \circ \Phi_{1}, H_{k_{1}}, H_{k_{2}}\right): \widetilde{\mathscr{B}}_{\hat{\varepsilon}} \cong \widetilde{\mathscr{B}}_{\hat{\varepsilon}} .
$$

Thus, the stretching along the paths property is proved, and by Theorem 2.1, we get the result.

Remark 4.1 The argument of the proof works the same (with minor modifications) if we replace the Beddington-DeAngelis term $\frac{x_{2}}{1+x_{3}}$ in Eq. (4.3) with a term of the form $x_{2} k\left(x_{3}\right)$ where $k(\cdot)$ is a positive and strictly monotone function defined on the positive real numbers.

\subsection{Proof of Theorem 3.1}

The proof of our result follows from the above construction and using Corollary 2.1. To this aim, take as $K_{0}$ and $K_{1}$ the following sets

$$
\tilde{H}_{k_{1}}:=\left\{q \in H_{k_{1}}: \Phi^{1}(q) \in \mathscr{B}_{\hat{\epsilon}}, \quad k_{*} \leq \mathscr{F}\left(\Phi_{1}^{1}(q), \Phi_{3}^{1}(q)\right) \leq k^{*}\right\}
$$

and

$$
\tilde{H}_{k_{2}}:=\left\{q \in H_{k_{2}}: \Phi^{1}(q) \in \mathscr{B}_{\hat{\epsilon}}, \quad k_{*} \leq \mathscr{F}\left(\Phi_{1}^{1}(q), \Phi_{3}^{1}(q)\right) \leq k^{*}\right\},
$$

which are slight modifications of the compact sets $H_{k_{1}}$ and $H_{k_{2}}$ introduced in the proof of Proposition 3.1.

\section{Discussion}

We conclude this paper with a brief discussion about the results obtained and their biological meaning.

The purpose of our work has been to prove analytically the presence of chaotic dynamics in a broad class of 3D systems of predator-prey type. More precisely, under certain conditions, we are able to determine explicitly a bounded region $\mathcal{R}$ containing two disjoint sets $K_{0}$ and $K_{1}$ satisfying the coin tossing property with respect to the Poincaré map $\Phi$. With this notion we mean that, given an arbitrary two-sided sequence of symbols $\left(s_{n}\right)_{n \in \mathbb{Z}} \in\{0,1\}^{\mathbb{Z}}$, there exists at least one point $q \in \mathcal{R}$ such that the full orbit $\mathscr{O}(q)$ along $q$ reproduces the behavior of the prescribed sequence. In order to give a hint of our concept, let us restrict to 
forward sequences and take, for instance, the sequences $010010001 \ldots$ and $001001001 \ldots$ Associated to such sequences we have two points $w, z \in \mathcal{R}$ with the following properties:

$$
\begin{aligned}
& w \in K_{0}, \Phi(w) \in K_{1}, \Phi^{2}(w) \in K_{0}, \Phi^{3}(w) \in K_{0}, \Phi^{4}(w) \in K_{1} \ldots \\
& z \in K_{0}, \Phi(z) \in K_{0}, \Phi^{2}(z) \in K_{1}, \Phi^{3}(z) \in K_{0}, \Phi^{4}(z) \in K_{0} \ldots
\end{aligned}
$$

If the sequence of symbols is periodic, we can find a corresponding periodic point for $\Phi$ as well.

From the point of view of the applications, the presence of regions containing such chaotic sets is quite relevant since in these sets we have phenomena of sensitive dependence. This fact makes impossible to predict the dynamics in the future from experimental data since small errors can cause great changes in long-time behavior.

Acknowledgments The authors thank the two referees for their interesting remarks and comments about the preliminary version of this article. The first author acknowledges the kind hospitality of the Department of Mathematics and Computer Science of the University of Udine. Alfonso Ruiz-Herrera was supported by the research project MTM 2008-02502, Ministerio de Educación y Ciencia, Spain. Fabio Zanolin was supported by the Project MIUR-PRIN “Ordinary Differential Equations and Applications”.

\section{References}

1. Arioli, G., Zgliczyński, P.: Symbolic dynamics for the Hénon-Heiles Hamiltonian on the critical level. J. Differ. Equ. 171, 173-202 (2001)

2. Arnold, V.I., Kozlov, V.V., Neishtadt, A.I.: Mathematical Aspects of Classical and Celestial Mechanics. Dynamical Systems III, Encyclopaedia Math. Sci., vol. 3. Springer, Berlin (1993)

3. Aulbach, B., Kieninger, B.: On three definitions of chaos. Nonlinear Dyn. Syst. Theory 1, 23-37 (2001)

4. Battelli, F., Palmer, K.J.: Singular perturbations, transversality, and Sil'nikov saddle-focus homoclinic orbits. J. Dyn. Differ. Equ. 15, 357-425 (2003)

5. Beddington, J.R.: Mutual interference between parasites or predators and its effect on searching efficiency. J. Animal Ecol. 44, 331-340 (1975)

6. Burns, K., Weiss, H.: A geometric criterion for positive topological entropy. Comm. Math. Phys. 172, 95-118 (1995)

7. Burton, R., Easton, R.W.: Ergodicity of linked twist maps. Global theory of dynamical systems. In: Proceedings of the International Conference, Northwestern University, Evanston, Ill., 1979. Lecture Notes in Math., vol. 819, pp. 35-49. Springer, Berlin (1980)

8. Capietto, A., Dambrosio, W., Papini, D.: Superlinear indefinite equations on the real line and chaotic dynamics. J. Differ. Equ. 181, 419-438 (2002)

9. Carbinatto, M., Kwapisz, J., Mischaikow, K.: Horseshoes and the Conley index spectrum. Ergod. Theory Dyn. Syst. 20, 365-377 (2000)

10. Cui, J., Takeuchi, Y.: Permanence, extinction and periodic solution of predator-prey system with Beddington-DeAngelis functional response. J. Math. Anal. Appl. 317, 464-474 (2006)

11. DeAngelis, D.L., Goldstein, R.A., O’Neill, R.V.: A model for trophic interaction. Ecology 56, 881-892 (1975)

12. Devaney, R.L.: Subshifts of finite type in linked twist mappings. Proc. Am. Math. Soc. 71, 334-338 (1978)

13. DuBowy, P.J.: Waterfowl communities and seasonal environments: temporal variability in interspecific competition. Ecology 69, 1439-1453 (1988)

14. Guckenheimer, J., Holmes, P.: Nonlinear Oscillations, Dynamical Systems, and Bifurcations of Vector Fields, Revised and Corrected Reprint of the 1983 Original. Applied Mathematical Sciences, vol. 42. Springer, New York, NY (1990)

15. Hsu, S.-B., Zhao, X.-Q.: A Lotka-Volterra competition model with seasonal succession. J. Math. Biol. 64, 109-130 (2012)

16. Hu, S.S., Tessier, A.J.: Seasonal succession and the strength of intra- and interspecific competition in a Daphnia assemblage. Ecology 76, 2278-2294 (1995)

17. Huppert, A., Blasius, B., Olinky, R., Stone, L.: A model for seasonal phytoplankton blooms. J. Theoret. Biol. 236, 276-290 (2005) 
18. Keeling, M., Rohani, P., Grenfell, B.T.: Seasonally forced disease dynamics explored as switching between attractors. Phys. D 148, 317-335 (2001)

19. Kennedy, J., Koçak, S., Yorke, J.A.: A chaos lemma. Am. Math. Mon. 108, 411-423 (2001)

20. Kennedy, J., Yorke, J.A.: Topological horseshoes. Trans. Am. Math. Soc. 353, 2513-2530 (2001)

21. Kennedy, J., Yorke, J.A.: Generalized Hénon difference equations with delay. Univ. Iagel. Acta Math. 41, 9-28 (2003)

22. Kirchgraber, U., Stoffer, D.: On the definition of chaos. Z. Angew. Math. Mech. 69, 175-185 (1989)

23. Leray, J., Schauder, J.: Topologie et équations fonctionelles. Ann. Sci. École Norm. Sup. 51(3), 4578 (1934)

24. Malik, T., Smith, H.L.: Does dormancy increase fitness of bacterial populations in time-varying environments? Bull. Math. Biol. 70, 1140-1162 (2008)

25. Margheri, A., Rebelo, C., Zanolin, F.: Chaos in periodically perturbed planar Hamiltonian systems using linked twist maps. J. Differ. Equ. 249, 3233-3257 (2010)

26. Mawhin, J.: Leray-Schauder degree: a half century of extensions and applications. Topol. Methods Nonlinear Anal. 14, 195-228 (1999)

27. Medio, A., Pireddu, M., Zanolin, F.: Chaotic dynamics for maps in one and two dimensions: a geometrical method and applications to economics. Int. J. Bifur. Chaos Appl. Sci. Eng. 19, 3283-3309 (2009)

28. Moser, J.: Stable and random motions in dynamical systems. With special emphasis on celestial mechanics, Hermann Weyl Lectures, the Institute for Advanced Study, Princeton, NJ. Annals of Mathematics Studies, no. 77. Princeton University Press, Princeton, NJ (1973)

29. Naji, R.K., Balasim, A.T.: On the dynamical behavior of three species food web model. Chaos Solitons Fractals 34, 1636-1648 (2007)

30. Palmer, K.J.: Exponential Dichotomies, the Shadowing Lemma and Transversal Homoclinic Points. Dynamics reported, vol. 1, pp. 265-306, Dynam. Report. Ser. Dynam. Systems Appl., 1. Wiley, Chichester (1988)

31. Pang, P.Y.H., Wang, M.: Strategy and stationary pattern in a three-species predator-prey model. J. Differ. Equ. 200, 245-273 (2004)

32. Papini, D., Zanolin, F.: On the periodic boundary value problem and chaotic-like dynamics for nonlinear Hill's equations. Adv. Nonlinear Stud. 4, 71-91 (2004)

33. Pireddu, M., Zanolin, F.: Cutting surfaces and applications to periodic points and chaotic-like dynamics. Topol. Methods Nonlinear Anal. 30, 279-319 (2007). Correction in Topol. Methods Nonlinear Anal. 33, 395 (2009)

34. Przytycki, F.: Ergodicity of toral linked twist mappings. Ann. Sci. École Norm. Sup. 16(4), 345-354 (1983)

35. Robinson, C.: Dynamical Systems. Stability, Symbolic Dynamics, and Chaos, Second edition. Studies in Advanced Mathematics. CRC Press, Boca Raton, FL (1999)

36. Schreiber, S.: Coexistence for species sharing a predator. J. Differ. Equ. 196, 209-225 (2004)

37. Smale, S.: Diffeomorphisms with many periodic points. In: Differential and Combinatorial Topology (A Symposium in Honor of Marston Morse) pp. 63-80. Princeton University Press, Princeton, NJ (1965)

38. Smale, S.: Differentiable dynamical systems. Bull. Am. Math. Soc. 73, 747-817 (1967)

39. Springham, J.: Ergodic properties of linked-twist maps. PhD thesis, University of Bristol. Available at ArXiv:0812.0899v1. http://arxiv.org/abs/0812.0899v1

40. Springham, J., Wiggins, S.: Bernoulli linked-twist maps in the plane. Dyn. Syst. 25, 483-499 (2010)

41. Srzednicki, R., Wójcik, K., Zgliczyński, P.: Fixed point results based on the Ważewski method. In: Handbook of topological fixed point theory, pp. 905-943. Springer, Dordrecht (2005)

42. Sturman, R., Meier, S.W., Ottino, J.M., Wiggins, S.: Linked twist map formalism in two and three dimensions applied to mixing in tumbled granular flows. J. Fluid Mech. 602, 129-174 (2008)

43. Sturman, R., Ottino, J., Wiggins, S.: The Mathematical Foundations of Mixing. The Linked Twist Map as a Paradigm in Applications: Micro to Macro, Fluids to Solids, Cambridge Monographs on Applied and Computational Mathematics, vol. 22. Cambridge University Press, Cambridge (2006)

44. Waldvogel, J.: The period in the Lotka-Volterra system is monotonic. J. Math. Anal. Appl. 114, 178184 (1986)

45. Walters, P.: An Introduction to Ergodic Theory. Graduate Texts in Mathematics, vol. 79. Springer, New York, NY, Berlin (1982)

46. Wiggins, S.: Global Bifurcations and Chaos. Analytical Methods. Applied Mathematical Sciences, vol. 73. Springer, New York, NY (1988)

47. Wiggins, S., Ottino, J.M.: Foundations of chaotic mixing. Philos. Trans. R. Soc. Lond. Ser. A Math. Phys. Eng. Sci. 362, 937-970 (2004)

48. Wójcik, K.: Remark on complicated dynamics of some planar system. J. Math. Anal. Appl. 271, 257266 (2002) 
49. Wojtkowski, M.: Linked twist mappings have the K-property. In: Nonlinear Dynamics (International Conference, New York, 1979), pp. 65-76, Annals of the New York Academy Sciences, vol. 357. New York (1980)

50. Zgliczyński, P.: Fixed point index for iterations of maps, topological horseshoe and chaos. Topol. Methods Nonlinear Anal. 8, 169-177 (1996)

51. Zgliczyński, P., Gidea, M.: Covering relations for multidimensional dynamical systems. J. Differ. Equ. 202, 32-58 (2004) 